\section{Papel de los microRNAs en la enfermedad periodontal}

\section{Role of microRNAs in periodontal disease}

\section{Sr. Editor}

La enfermedad periodontal (EP) es un desorden inflamatorio e infeccioso que afecta las estructuras de soporte del diente, siendo el resultado de la interacción entre bacterias patógenas y el sistema inmune ${ }^{1}$, anteriormente se pensaba que esta enfermedad se presentaba de forma lineal en donde la gingivitis precedía a la EP y ésta conllevaba a la pérdida ósea, actualmente se ha demostrado que la distribución y severidad de la misma está fuertemente influenciada por la susceptibilidad del huésped ${ }^{2}$. Se estima que entre un 20 y $50 \%$ de la población mundial se ve afectada por $\mathrm{EP}^{3}$. En México son escasos los datos epidemiológicos que representen al país, sin embargo García-Pérez et al. ${ }^{4}$ determinaron la prevalencia de condiciones a nivel gingival y periodontal en Chiapas, México, reportando que el $68,5 \%$ de la población de estudio presentaba problemas gingivales y el $8,7 \%$ periodontitis, siendo afectados aquellos que vivían en zonas rurales. Por otra parte, Borjes-Yañes et al. ${ }^{5}$ desarrollaron un estudio en adultos mayores de 60 años $(\mathrm{N}=473)$ mencionando que el $28 \%$ de la población presentaba periodontitis moderada.

Los métodos diagnósticos actuales van orientados a dirigir el tratamiento en base a las características de los defectos óseos, o a las alteraciones en los tejidos blandos de soporte del diente, sin embargo, se han empezado a proponer métodos moleculares con la finalidad de que se pueda identificar el desarrollo de esta enfermedad antes de que llegue a provocar un daño en cualquiera de los tejidos de soporte del diente. Dentro de las moléculas que destacan en la literatura se encuentran los microRNAs (miRNAs); son ácidos nucleicos de expresión ubicua no codificantes que están formados por 18 a 25 nucleótidos de longitud, los cuales juegan un papel importante en la regulación post-transcripcional de la expresión génica ${ }^{6}$.

La expresión diferencial que se ha reportado de los miRNAs en los diferentes tipos de EP hasta la actualidad es poca y diversa; Xie et al. ${ }^{7}$ analizaron la expresión de los miRNAs en tejido gingival de pacientes con y sin EP, encontrando una sobre-expresión de diversos miRNAs (hsamiR-126, hsa-miR-20a, hsa-miR-142-3p, hsa-miR-19a, hsa-let-7f, hsamiR-203, hsa-miR-17, hsa-miR-223, hsa-miR-146b y hsa-miR-146a) y una sub expresión de hsa-miR-155 y hsa-miR-205; teniendo como genes blanco aquellos relacionados con procesos de inflamación, adherencia
ISSN: 1560-9111

\section{Cartas al Editor}

María Verónica Cuevas-González ${ }^{1, a}$,

Alma Graciela García-Calderón 2,b,

Dalia Abril Guzmán-Gastelum ${ }^{2, c}$,

Graciela Zambrano-Galván ${ }^{3, d}$,

Alejandro Donohue-Cornejo ${ }^{2, \mathrm{e}}$,

Juan Carlos Cuevas-González 2,e

${ }^{1}$ Universidad Nacional Autónoma de México.

Programa de Maestría y Doctorado en

Ciencias Médicas, Odontológicas y de la

Salud. Ciudad de México, México.

2 Universidad Autónoma de Ciudad Juárez.

Departamento de Estomatología. Ciudad

Juárez Chihuahua, México.

${ }^{3}$ Universidad Juárez del Estado de Durango.

Facultad de Odontología. Durango, Dgo.

México.

a Maestra en Ciencias.

${ }^{\mathrm{b}}$ Especialista en Periodoncia.

c Maestra en Ciencias.

d Doctora en Ciencias.

e Doctor en Ciencias.

Correspondencia

Juan Carlos Cuevas González

Correo electrónico: cuevas_gonzalez@hotmail.com Departamento de Estomatología Universidad

Autónoma de Ciudad Juárez Anillo Envolvente del Pronaf s/n, Zona Pronaf, 32315 Cd Juárez Chihuahua, México.

Coautores:

María Verónica Cuevas-González

cuevas_veronica@live.com.mx

Alma Graciela García-Calderón

alma.garcia@uacj.mx

Dalia Abril Guzmán-Gastelum

dguzman@uacj.mx

Graciela Zambrano-Galván

gzambrano@ujed.mx

Alejandro Donohue-Cornejo

adonohue@uacj.mx

Conflicto de intereses: Los autores declaran no tener conflictos de interés.

Fecha de recepción: 24/10/18

Fecha de aceptación: 26/11/18

(c) Los autores. Este artículo es publicado por la revista Odontología Sanmarquina de la Facultad de Odontología, Universidad Nacional Mayor de San Marcos. Este es un artículo de acceso abierto, distribuido bajo los términos de la licencia Creative Commons Atribucion - No Comercia_Compartir Igual 4.0 Internacional. (http://creativecommons.org/licenses/by-nc-sa/4.0/) que permite el uso no comercial, distribución y reproducción en cualquier medio, siempre que la obra original sea debidamente citada. 
a leucocitos, hiperplasia vascular, proliferación celular así como reabsorción ósea; por otra parte Lee et al. ${ }^{8}$ sugieren que múltiples miRNAs (miR-181b, miR-19b, miR-23a, miR-30a, miR-let7a y miR-301a) pueden jugar un papel en el desarrollo de la periodontitis crónica tras analizar tejido gingival.

De igual forma se han publicado estudios en los que se analiza la presencia de los mismos a partir de bio-fluidos; Saito et al. ${ }^{9}$ en un estudio piloto determinaron la presencia de miRNAs en muestras de fluido crevicular de pacientes con EP y sujetos libres de ella identificando que hsa-miR-451a, hsa-miR-223-3p, hsa-miR-486-5p, hsa-miR-1260a, hsa-miR-203a, hsa-miR-210-3p y hsa-miR-205-3p se encontraban diferencialmente expresados entre ambos grupos; de la misma forma, Micó-Martínez et al. ${ }^{10}$ en un estudio realizado a partir de muestras de fluido crevicular gingival, encontraron diferencias significativas de miR-1226 ente sujetos con periodontitis crónica y sujetos libres de EP. Debido a la información que se encuentra publicada hasta el momento y al potencial que representa esta línea de investigación, decidimos realizar esta carta al editor con el objetivo de compartir parte de la información disponible, favorecer un mayor entendimiento acerca de la participación de estas moléculas en la EP, y desarrollar en un futuro proyectos en el área de la periodoncia que puedan ser aplicados en este grupo de pacientes.

Los métodos diagnósticos de la EP son un punto medular en el tratamiento oportuno de esta enfermedad, las investigaciones recientes van un paso más allá, y están orientadas hacia la identificación de blancos moleculares que prevengan el desarrollo de la misma, los miRNAs son moléculas que debido a su función reguladora cobran especial interés en la patogénesis de este padecimiento, si bien diversos autores han propuesto distintos papeles de miRNAs que pueden emplearse como marcadores entre los distintos tipos de EP, aún falta validarlos en poblaciones más grandes que permitan la aplicabilidad de los mismos.

\section{Rereferencias bibliográficas}

1. Martínez-Herrera M, Silvestre-Rangil J, Silvestre FJ. Association between obesity and periodontal disease. A systematic review of epidemiological studies and controlled clinical trials. Med Oral Patol Oral Cir Bucal. 2017;22(6):708-15.

2. Costa FO, Susin C, Cortelli JR, Almeida Pordeus I. Epidemiology of periodontal disease. Int J Dent. 2012:848641. doi: 10.1155/2012/848641.

3. Nazir MA. Prevalence of periodontal disease, its association with systemic diseases and prevention. Int J Health Sci (Qassim). 2017;11(2):72-80.

4. García-Pérez Á, Borges-Yáñez SA, Jiménez-Corona A, Jiménez-Corona ME, Ponce-de-León S. Self-report of gingival problems and periodontitis in indigenous and non-indigenous populations in Chiapas, Mexico. Int Dent J. 2016;66(2):105-12.

5. Borges-Yáñez SA, Irigoyen-Camacho ME, Maupomé G. Risk factors and prevalence of periodontitis in community-dwelling elders in Mexico. J Clin Periodontol. 2006;33(3):184-94.

6. Lin S, Gregory RI. MicroRNA biogenesis pathways in cancer. Nat Rev Cancer. 2015;15(6):321-33.

7. Xie YF, Shu R, Jiang SY, Liu DL, Zhang XL. Comparison of microRNA profiles of human periodontal disease and healthy gingival tissues. Int J Oral Sci. 2011;3(3):125-34.

8. Lee YH, Na HS, Jeong SY, Jeong SH, Park HR, Chung J. Comparison of inflammatory microRNA expression in healthy and periodontitis tissues. Biocell. 2011;35(2):43-9.

9. Saito A, Horie M, Ejiri K, Aoki A, Katagiri S, Maekawa $S$, et al. MicroRNA profiling in gingival crevicular fluid of periodontitis-a pilot study. FEBS Open Bio. 2017; 7(7):981-94

10. Micó-Martínez P, García-Giménez JL, Seco-Cervera M, López-Roldán A, Almiñana-Pastor PJ, Alpiste-Illueca F, et al. miR-1226 detection in GCF as potential biomarker of chronic periodontitis: A pilot study. Med Oral Patol Oral Cir Bucal. 2018;23(3):308-14. 